\title{
Globe
}

Revue internationale d'études québécoises

\section{David Hackett Fischer, Le rêve de Champlain, Sillery, Septentrion, 2011}

\section{John A. Dickinson}

Volume 15, numéro 1-2, 2012

URI : https://id.erudit.org/iderudit/1014641ar

DOI : https://doi.org/10.7202/1014641ar

Aller au sommaire du numéro

\section{Éditeur(s)}

Globe, Revue internationale d'études québécoises

\section{ISSN}

1481-5869 (imprimé)

1923-8231 (numérique)

Découvrir la revue

Citer ce compte rendu

Dickinson, J. A. (2012). Compte rendu de [David Hackett Fischer, Le rêve de Champlain, Sillery, Septentrion, 2011]. Globe, 15(1-2), 346-349.

https://doi.org/10.7202/1014641ar d'utilisation que vous pouvez consulter en ligne.

https://apropos.erudit.org/fr/usagers/politique-dutilisation/ 
confrontation et de concertation située aux frontières entre les principales communautés de la ville. Même les évènements les plus inclusifs, les fêtes civiques par exemple, sont conçus selon les auteures de manière à ce que la "différence» soit reconnue (p. 327).

Voici donc un livre important, imposant avec ses 500 pages et plus, illustré abondamment avec de magnifiques photographies et gravures de l'époque, écrit dans un anglais à la fois élégant, précis et coloré, et qui témoigne amplement de la grande générosité d'esprit des deux auteures. Peut-on dire d'un livre que sa plus belle qualité est la générosité? Dans le cas présent, je suis prêt à l'affirmer, vu la taille, la valeur et l'intelligence du cadeau que Sherry Olson et Patricia Thornton viennent d'offrir à ceux et celles qui s'intéressent à l'histoire de Montréal.

Peter Gossage Université Concordia

\section{David Hackett Fischer \\ Le rêve de Champlain, Sillery, Septentrion, 2011.}

Les commémorations des premiers établissements français en Amérique du Nord ont donné lieu à une pléthore d'ouvrages sur les fondateurs et leur époque. On pourrait presque dire heureusement, car sans ces fêtes mémorielles, la Nouvelle-France serait inaperçue dans le tourbillon des écrits qui se focalisent sur un passé beaucoup plus récent. Elles servent certes à réveiller la mémoire, mais contribuent-elles vraiment à renouveler les problématiques et la connaissance historique ? C'est, me semble-t-il, la principale question qu'on doit se poser en abordant un ouvrage à caractère scientifique pour un compte rendu dans une revue universitaire.

Le Champlain de David Hackett Fischer est un pavé volumineux, marqué par une grande érudition, un style enlevant et qui se veut sans doute la biographie "définitive» de Champlain. La biographie conventionnelle est complétée par un essai sur la mémoire de Champlain et seize "appendices" (en français, on privilégierait "annexes»), pas toujours indispensables, sur une grande variété de sujets allant de la date de naissance aux mesures utilisées à l'époque. L'auteur a beaucoup lu sur son sujet et on ne peut lui reprocher de ne pas bien connaître la littérature scientifique sur la France du 
tournant du XVI ${ }^{\mathrm{e}}$ siècle. Comme les sources sur cette période sont limitées, les écrits de Champlain constituent l'ossature essentielle de l'ouvrage, ce qui est tout à fait normal. L'auteur n'a pas réussi à trouver de nouvelles sources qui jetteraient une nouvelle lumière sur ce personnage incontournable des origines du Canada, mais il a intégré des détails que les autres biographes de Champlain ne connaissaient pas ou qu'ils avaient tenus pour moins importants. En ce sens, c'est la biographie la plus complète du fondateur de Québec.

On sait peu de choses sur Samuel de Champlain avant 1603. Sa date de naissance demeure un sujet de discussion; sa religion dans sa jeunesse et l'éducation reçue restent des points ambivalents; son voyage dans les Antilles est contesté. Cependant, à partir de 1603, nous disposons des écrits du principal protagoniste, qui ne manque pas de se mettre en valeur. Compagnon du sieur de Monts et de Pont-Gravé en Acadie, il contribue à jeter les bases d'une présence française sur la côte atlantique. À la suite de l'abandon de ce projet, il commande à Québec pendant l'hiver calamiteux de 16081609. Cette expérience fixe son destin ; désormais, c’est la vallée du SaintLaurent qui sera au cœur de ses activités. Entre la recherche d'appuis à la cour des Bourbons, de soutiens financiers dans les milieux marchands de l'Ouest français et d'accommodements avec les nations amérindiennes de la Nouvelle-France, il passera un quart de siècle à œuvrer pour tenter d'assurer une emprise française sur le territoire et sur ses habitants.

L'histoire de Champlain est bien connue de tous ceux qui fréquentent tant soit peu les études québécoises : alliances avec les nations du Saint-Laurent et des Hurons qui l'opposent aux Iroquois au sud, notamment au cours de trois batailles $(1609,1610,1615)$; voyages et cartographie de vastes régions de l'Amérique du Nord-Est qui permettent de dresser des cartes magnifiques de ces territoires; va-et-vient entre l'Europe et l'Amérique pendant une trentaine d'années qui le met en contact avec les puissances en France et dans le milieu amérindien; négociations ardues avec des marchands protestants, des missionnaires catholiques et des intérêts privés et publics qui soulignent sa capacité à trouver des compromis (qualité bien canadienne). Champlain est un homme qui a marqué les origines du Canada et qui mérite notre respect. Mais faut-il l'élever en quasi-saint et ne pas envisager des hypothèses moins favorables au « héros» Champlain?

L'auteur connaît bien les sources et l'historiographie, mais il conclut toujours en faveur de son héros. Certes, les scénarios qu’il monte sont possibles, mais d'autres moins positifs le sont aussi. C'est ce qui agace 
un historien qui estime que les preuves formelles méritent plus que des suppositions sans fondements. Où commencer?

Au début de ce récit, l'auteur affirme que la seule image de Champlain par lui-même est la gravure du combat en 1609. Or il s'agit d'une gravure qui est d'une autre main que celle de Champlain (à moins que Champlain ne fût graveur?) et qui comporte des détails problématiques: les arbres qui ressemblent à des palmiers, les canots qui n'ont rien à voir avec l'Amérique du Nord et les guerriers nus quand toutes les autres sources (même Champlain) les décrivent vêtus d'armures de bois. Fischer condamne ses prédécesseurs pour leurs descriptions farfelues, mais la sienne est-elle plus réaliste? Le maître mot de Fischer est "probablement ", ce qui signifie qu'il n'en sait rien, mais qu'il laisse libre cours à son imagination. Pour un historien rigoureux, ce n'est pas acceptable, quitte à passer pour un iconoclaste ou un révisionniste (termes qu'affectionne l'auteur dès qu'on émet la moindre critique du héros)! Sur sa jeunesse, on aurait avantage à consulter l'introduction de la dernière édition de son voyage de 1603 de Conrad E. Heidenreich et $\mathrm{K}$. Janet Ritch ${ }^{1}$, plus sobre et plus crédible.

Il est juste de dire que l'auteur évalue toutes les remarques qui enfoncent le héros, mais aussi qu'il accepte toujours les affirmations de Champlain sans esprit critique. Il conclut toujours que celui-ci a raison contre tous. Ce n'est pas une démarche d'historien scientifique malgré l'intérêt qu'elle a pour une histoire populaire valorisant les grands hommes. En fait, c'est une histoire qui plaît aux masses (ce qui n'est pas le fait de la plupart des synthèses), mais ajoute-t-elle quelque chose à notre compréhension du passé ? Voilà la question fondamentale.

Malheureusement, cette hagiographie de Champlain n'ajoute rien de concret. Les "nouveautés » ne sont que des conjectures sans preuve issues de l'imagination fertile de l'auteur. Champlain (roturier d'origine modeste) «ami » des grands, dont Henri IV, ce n'est tout simplement pas crédible. L'auteur met en scène un surhomme qui n'a jamais existé et obscurcit un homme plus humble et plus réaliste qui a fait beaucoup de choses importantes.

En définitive, cette biographie (hagiographie) de Champlain relève plus du roman que de l'histoire, notamment pour les périodes pour lesquelles l'on ne dispose pas de sources. Les nostalgiques d'une histoire dominée par les grands hommes d'origine européenne seront ravis; les

$$
4+4
$$

1. Conrad E. Heidenreich et K. Janet Ritch, Samuel de Champlain before 1604. Des Sauvages and Other Documents Related to the Period, Toronto, The Champlain Society, 2010. 
historiens plus rigoureux resteront sceptiques devant tant d'affirmations gratuites portant sur un Champlain rêvé et héroïque plus grand que nature.

John A. Dickinson

Université de Montréal

\section{Frédéric Smith}

"La France appelle votre secours». Québec et la France libre 1940-1945, Montréal, VLB éditeur, 2012.

Sous un titre tiré pour partie du vibrant appel lancé par le général de Gaulle aux Canadiens français le $1^{\text {er }}$ août 1940, Frédéric Smith nous propose de revisiter la question de l'implantation et de la diffusion de la France libre au Québec.

Pour ce faire, il utilise une bibliographie maîtrisée, mais qui fait l'impasse sur certains travaux universitaires non publiés. On peut penser ici aux mémoires de maîtrise de Stéphane Dionne, de Jenny-Louise Sexton et de Samuel Trudeau, ou aux thèses de doctorat en histoire de Sylvie Guillaume (Bordeaux III, 1975) et d'Olivier Courteaux (Paris IV, 2000). Smith emploie également des sources primaires originales, en particulier les fonds Auguste Viatte ou Marthe Simard. Utilement illustré, l'ensemble de l'ouvrage est agréable à lire, et propose une édition de bonne facture.

Dans la continuité des travaux existants, notamment ceux d'Éric Amyot, l'auteur confirme ce que les historiens martèlent depuis longtemps: le Québec ne fut ni monolithique ni figé dans son attitude face à Vichy et à la France libre, n'en déplaise aux tenants d'une mémoire hémiplégique! Ainsi, il souligne bien les clivages qui divisent la société québécoise, les vecteurs d'opinion (la presse), les élites et l'Église, au sein de laquelle le Cardinal Villeneuve aurait, dès 1941 selon l'auteur, opéré une discrète surveillance, voire une "épuration " des éléments "les plus ardemment vichystes" (p. 107) : une piste qui mériterait assurément d'être creusée. De même, Smith confirme la nécessité de se doter d'une chronologie fine pour aborder une opinion progaulliste ici comme ailleurs (cf. les travaux de Pierre Laborie en France) très évolutive: de la réticence ou de l'indifférence initiale aux premiers infléchissements de 1941, jusqu'au retournement majoritaire de 1943. Plus encore, il montre la somme des difficultés rencontrées et in fine surmontées. 